\title{
¿QUiÉn ESTÁ EN CONTRA DE LA INMIGRACIÓN EN LA ARGENTINA CONTEMPORÁNEA? INTERESES Y VALORES EN LAS ACTITUDES DE LA POBLACIÓN HACIA LA INMIGRACIÓN
}

\author{
Guillermo Cantor ${ }^{1}$
}

Resumen: Los cambios demográficos provocados por flujos migratorios recientes en la Argentina han dado lugar a diversas actitudes y prácticas discriminatorias y/o xenófobas que frecuentemente son reportadas en la prensa y en trabajos académicos. Sin embargo, no está claro en qué medida estas actitudes negativas reflejan una percepción difundida en la población y, menos aún, en qué se fundan las mismas. El presente trabajo indaga en las percepciones que los argentinos tienen respecto de la política inmigratoria y, por ende, de la inmigración. Basándome en datos de la Encuesta Mundial de Valores, encuentro que el rechazo a las políticas inmigratorias abiertas no parece estar basado en motivaciones de tipo económica, como suele ocurrir en otros países. Por el contrario, tal como se desprende del análisis aquí presentado, dicho rechazo parece estar más asociado a factores culturales, ideológicos, psicosociales y de confianza.

Abstract: In Argentina, demographic changes prompted by recent migratory flows are often followed by various types of discriminatory and xenophobic attitudes and practices. These sentiments are routinely reported both in the media and in academic papers. However, it is not clear the extent to which these negative attitudes reflect a widespread perception in the population at large. Even less

Guillermo Cantor es Doctor en Sociología por la Universidad de Maryland (Estados Unidos) y actualmente se desempeña como Investigador Asistente en el Consejo de Investigaciones Científicas y Técnicas de Argentina. Email: guillermocantor@conicet.gov.ar. 
apparent are the factors that explain them. This article examines the attitudes that Argentinians have regarding immigration policy and immigration. Based on data from the World Values Survey, I find that unlike what commonly occurs in other countries, rejection to open immigration policies in Argentina does not seem to be based on economic motivations. On the contrary, according to the analysis presented in this article, such rejection seems to be more closely associated with cultural, ideological, psychosocial and trust-related factors.

\section{Introducción}

La Argentina es recurrentemente caracterizada como un país de inmigrantes. En gran medida, ese calificativo deriva del impresionante flujo migratorio que llegó al país, principalmente desde Europa, desde mediados del siglo XIX hasta mediados del XX. En base a este gran fenómeno migratorio que cambió de manera drástica la composición demográfica del país, se ha ido forjando un discurso bastante difundido que asigna un valor positivo a la llegada de estas olas de inmigrantes y la contribución que estos nuevos pobladores han realizado al desarrollo del país. A mediados del siglo XX, sin embargo, la Argentina pierde preeminencia como meta para los inmigrantes europeos, mientras que sigue siendo un destino importante para los migrantes que provienen de países vecinos (Pacecca y Courtis, 2008). Como consecuencia de ello, el flujo migratorio dirigido hacia el país cambia su composición. Mientras que en las primeras décadas del siglo XX la gran mayoría de la población nacida en el extranjero era de origen europeo, a comienzos del siglo XXI la mayor parte de los inmigrantes residentes en la Argentina proviene de países vecinos. Puntualmente, de acuerdo a datos del Censo de Población y Vivienda, en 1914 los inmigrantes procedentes de países no limítrofes -especialmente de 
países europeos- representaban el 93\% de la población extranjera residente en el país; en 2001, en cambio, $60 \%$ de la población nacida en el extranjero era originaria de países limítrofes.

Los cambios demográficos provocados por los flujos migratorios recientes han dado lugar a diversas actitudes y prácticas discriminatorias y/o xenófobas que ocasionalmente son reportadas en la prensa y en trabajos académicos. Es decir, a diferencia de la alta valoración que tradicionalmente se ha asignado a la inmigración procedente de los países europeos, la inmigración procedente de países vecinos es frecuentemente caracterizada como problema o bien abiertamente denostada. Sin embargo, no está claro en qué medida estas actitudes negativas reflejan una percepción difundida en la población y, menos aún, en qué se fundan las mismas.

En los países industrializados, la integración de inmigrantes que llegan a sus tierras ha sido objeto de fuerte preocupación tanto por parte de los gobiernos como de los académicos que estudian el tema de la inmigración. En ese sentido, la receptividad que las poblaciones de los países de destino tienen respecto de los recién llegados ha dado lugar a numerosos estudios en dichos países. Ahora bien, ¿ por qué es importante prestar atención a la opinión pública sobre la inmigración y los factores que influyen en las actitudes públicas sobre esta cuestión? Entre las razones que se suelen invocar se destacan: (1) el hecho de que en sistemas democráticos supuestamente las actitudes públicas de los votantes influyen en las políticas públicas que se adoptan (O'Rourke y Sinnott, 2006; Esses, Dovidio y Hodson, 2002; Scheve y Slaughter, 2001); (2) el impacto que las actitudes respecto a la inmigración tienen en el comportamiento cotidiano de los individuos y, por ende, en el clima social general de la nación receptora (Esses, Dovidio y Hodson, 2002; Berg, 2009; Vala, Pereira y Ramos, 
2006), y (3) la influencia directa que dichas actitudes tienen sobre los flujos de migración internacional (Mayda, 2004).

En la Argentina, por su parte, se registran menos estudios sistemáticos sobre este tema y los mismos suelen estar orientados más a la reconstrucción de discursos discriminatorios que circulan tanto desde diferentes agencias del Estado como en el accionar de organizaciones de diverso tipo y entre la población en general. Poco se sabe, sin embargo, sobre los motivos que llevan a la población a tener posiciones de mayor o menor aceptación respecto a los migrantes. En este marco, el presente trabajo se propone indagar en las percepciones que los argentinos tienen respecto de la política inmigratoria e, indirectamente, de los inmigrantes. La pregunta que guía el presente estudio es ¿cuáles son los factores que llevan a los argentinos a tener una actitud más o menos tolerante respecto de la inmigración? Para ello, en este trabajo se explora el comportamiento de factores de índole económica y no económica. En particular, me interesa analizar el rol que la confianza respecto de terceros desconocidos tiene respecto de la apertura del país a la llegada a nuevos migrantes.

Basándome en datos de la Encuesta Mundial de Valores (EMV), ${ }^{2}$ utilizo análisis multivariados para evaluar el impacto que la situación económica individual, factores culturales, el posicionamiento ideológico, la satisfacción con la vida, y la confianza respecto de otros tiene en las actitudes de la población respecto de la política inmigratoria. Controlando por otras variables, los resultados demuestran que en contraposición con lo que señalan las

Los datos analizados corresponden a la onda 2006 de la EMV. En ese año, el tema migratorio cobró cierta notoriedad en la Argentina luego del incendio del taller textil de Luis Viale, con la consecuente muerte de seis muertos de nacionalidad boliviana. Por otra parte, en 2006 también se pone en vigencia el programa de regularización de inmigrantes llamado "Plan Patria Grande". 
interpretaciones que basan el rechazo a la inmigración en una percepción de competencia en el plano económico, esa hipótesis no tiene sustento empírico en el caso analizado. Por otra parte, el trabajo muestra que niveles altos de capital social (entendido en términos de confianza) facilitan la integración de inmigrantes en la medida en que aquellos ciudadanos que reportan tener más confianza hacia los otros tienden a mostrar actitudes más positivas hacia la inmigración. Al mismo tiempo, la tolerancia hacia la diferencia, las actitudes ideológicas más progresistas y el bienestar subjetivo también aparecen como positivamente asociados a las actitudes más abiertas hacia la inmigración.

\section{Actitudes hacia los migrantes en la Argentina}

Tal como se señalara más arriba, en la Argentina los trabajos sobre actitudes de la población nativa respecto de los inmigrantes recientes se han centrado mayormente en la descripción de las prácticas y discursos discriminatorios y xenófobos. En ese sentido, la literatura se ha enfocado tanto en el origen como en el contenido de esos discursos y prácticas (ver, por ejemplo, Oteiza, Novick y Aruj, 2000; Grimson, 2006 y 2005; Monner Sans, 2005; Nadali, 2007; Joseph, 2000; Gallinati 2008).

En un sentido general, la política de relaciones étnicas vigente en la Argentina desde su formación como nación se inscribe en el marco de un relato que concibe a la cultura nacional argentina como un crisol de razas (Grimson, 2005). En ese contexto, mientras se glorifica la inmigración europea, la inmigración contemporánea es mirada con sospecha cuando no directamente repudiada. Esta visión viene de la mano de la negación de "la raza" en la Argentina (Joseph, 2000). En su trabajo con porteños de clase media, Joseph interpreta las afirmaciones que encuentra sobre la 
supuesta no importancia de la categoría raza como evidencia del lugar relevante que la raza tiene en la identidad nacional. En otras palabras, existen conceptos específicos de raza que forman parte indisoluble del sentido que los porteños de clase media tienen sobre sí mismos y sobre la identidad nacional. Como blancos de esta parte del mundo, siguiendo el argumento de Joseph, los porteños imaginan su blancura en relación a los blancos de otras partes del mundo, su comunidad imaginaria cruza fronteras y traza límites de exclusión dentro de la misma nación.

Asimismo, si bien las naciones son construidas como artefactos culturales por los Estados a través de políticas y relatos desarrollados por los gobiernos, también es preciso observar visiones que circulan entre personas comunes y corrientes (Grimson, 2005). Consecuentemente, se señala la necesidad de recuperar las formas en las que los migrantes construyen sus propias identidades y los modos en que se relacionan con la sociedad mayor, a través de una recuperación de los relatos que en la vida cotidiana y a través de los medios de comunicación realizan los migrantes bolivianos residentes en Buenos Aires.

En cuanto a las fuentes de los discursos migratorios y xenófobos en períodos históricos recientes, el grueso de la atención analítica se ha enfocado en los discursos y prácticas puestos en acto por el mismo Estado a través de diferentes niveles y en diferentes gobiernos. En ese sentido, el trabajo de Grimson (2006) ilustra cómo durante los años '90 el gobierno argentino y los medios de comunicación acuñan interpretaciones distorsivas sobre un supuesto nuevo fenómeno inmigratorio que afectaba a la Argentina, según las cuales los inmigrantes serían responsables de los graves problemas de desocupación e inseguridad. La estigmatización de inmigrantes limítrofes se plasma en declaraciones de diferentes funcionarios del gobierno y en propuestas de legislación desarrolladas en ese período. 
En sintonía con esta línea, Oteiza, Novick y Aruj (2000) dan cuenta de cómo el Estado argentino a través de sus diferentes niveles ha ido construyendo un discurso para incriminar a los inmigrantes limítrofes por diferentes problemas económicos, sociales, sanitarios y de seguridad.

Con un enfoque centrado en lo local, que simultáneamente aborda los discursos del Estado pero también los que circulan en la sociedad civil, Nadali (2007) examina tanto los procesos de estigmatización de inmigrantes limítrofes (en particular, de paraguayos, chilenos y bolivianos) como las consecuentes prácticas de manipulación a los que son sometidos. Para ello, analiza el discurso de políticos, funcionarios, pobladores de zonas de frontera y la experiencia personal de los mismos inmigrantes. Como resultado, el trabajo no sólo da cuenta de los procesos de construcción de los estigmas, sino también de los lenguajes de réplicas a través de los cuales los inmigrantes deshacen y rehacen sus identidades. En particular, la autora también hace hincapié en los estereotipos sobre los inmigrantes desarrollados desde el mismo gobierno durante los ‘90, en los cuales se los asocia con connotaciones negativas tales como “'gran cantidad, 'peligrosidad, 'problema, 'invasión', 'diferencias culturales,' 'raza inferior”' (Nadali, 2007: 142). Asimismo, las acciones racistas o discriminatorias son producto de las representaciones y prácticas sociopolíticas desarrolladas por agentes socialmente significativos en determinados contextos y relaciones de poder. En ese sentido, tanto el poder político y sus instituciones como los medios de comunicación y los informes de expertos son generadores de estereotipos sobre los migrantes. En una línea similar, a través de un estudio del lenguaje, Monner Sans (2005) indaga acerca del tratamiento de la diversidad en la escuela, los medios de comunicación y la política en diversos contextos socio-geográficos del país. En particular, se destaca el ejercicio de poder a través de clasificaciones 
de diversos individuos o grupos que se construyen en espacios y tiempos anteriores a las situaciones analizadas.

En contraposición con la visión estatal excluyente y hostil hacia los inmigrantes limítrofes prevaleciente en la década de los noventa, Gallinati (2008) observa cómo el Estado argentino construye narrativas sobre su posicionamiento sobre la cuestión migratoria a partir de la implementación de una ley migratoria 25.871, promulgada en 2004. En esta línea, la cuestión migratoria es articulada con aquellas de los derechos humanos, la integración regional, el afianzamiento del sistema democrático en toda la región y el reconocimiento de los nuevos derechos entre otras.

Mirando desde abajo, en el universo discursivo que circula entre personas comunes, también la literatura ha reparado, aunque en menor medida, en prácticas discriminatorias y xenófobas. Hay una noción que sostiene que amplios sectores de la sociedad adhieren a la visión discriminadora encarnada por las instituciones estatales y los medios de comunicación (Grimson, 2006). Así, mirando en particular a la comunidad de bolivianos, Grimson (2005) repara en las dinámicas sociales de exclusión social y cultural, las cuales son percibidas por los inmigrantes bolivianos cotidianamente. Ellas abarcan desde la utilización de términos peyorativos, hasta acusaciones de robo infundadas, hasta formas de mirar "desde arriba" con desprecio, entre otras situaciones cotidianas. Sin embargo, en lugar de aparecer como objetos pasivos de la discriminación, los inmigrantes desarrollan estrategias en su relación cotidiana con diversos grupos socioculturales, con los cuales entran en conflicto o negocian sus identidades.

A nivel metodológico, la mayoría de los trabajos que dan cuenta de las actitudes respecto de los inmigrantes, suelen estar basados en enfoques etnográficos y orientados a identificar e interpretar las categorías frecuentemente utilizadas para referir a los migrantes. Sin embargo, poco se 
sabe sobre los factores que explicarían tales actitudes. Tal como señalé anteriormente, esto contrasta con la gran atención que el tema ha recibido en otros contextos geográficos.

\section{Explicaciones sobre las actitudes de la población hacia la inmigración}

Pasaré revista a continuación a algunas de las líneas argumentales más significativas presentes en la literatura comparada para dar cuenta de cómo determinadas características individuales de los sujetos afectan sus actitudes hacia los inmigrantes. ${ }^{3}$ Esta revisión servirá de punto de partida para el presente trabajo. A fin de sintetizar algunas de las líneas más relevantes encontradas en la literatura, las explicaciones fueron clasificadas en cuatro grupos, a saber: socioeconómicas, culturales, psico-sociales, y basadas en redes y en la confianza.

\section{Determinantes socioeconómicos}

Una línea dominante en la literatura comparada ha demostrado que la competencia económica entre grupos étnicos o "nacionales" produce actitudes de hostilidad y prejuicio. En base a esta línea argumental, las preferencias en relación con la inmigración se explican por motivaciones

Hay también un número considerable de investigaciones que para explicar las actitudes de los sujetos sobre la inmigración toman en cuenta variables contextuales, tales como: la riqueza del país de destino (Mayda, 2004); el rol de los medios de comunicación y los mensajes que estos transmiten sobre los inmigrantes (Boomgaarden y Vliegenthart, 2009); las políticas y prácticas inmigratorias y la situación económica de los países (Simon y Lynch, 1999), o la historia de las relaciones raciales en el país receptor (Diamond, 1998). Del mismo modo, Lupo (2010) pone el foco en la heterogeneidad cultural entre países y los regímenes de ciudadanía al analizar la relación entre inmigración y capital social. 
económicas de los individuos (como bien ilustran los trabajos de Burns y Gimpel, 2000; O’Rourke y Sinnott, 2006 y Zagefka et al., 2007). Puntualmente, aquellos segmentos de la población con menor calificación y educación tenderían a tener actitudes más anti-inmigratorias en virtud de que se sentirían más amenazados por los recién llegados (Scheve y Slaughter, 2001). En esta línea, por lo tanto, habría un auto-interés económico que determinaría las posiciones de la población sobre el tema inmigratorio.

Es sabido que una de las quejas más frecuentes con respecto a los inmigrantes -aunque no necesariamente fundadas en evidencia empírica- es que quitan fuentes de trabajo a los trabajadores nativos, contribuyen al crecimiento del desempleo, reducen los salarios, empeoran las condiciones de trabajo en algunas ocupaciones, y utilizan y disminuyen la calidad de los escasos recursos disponibles (Espenshade y Hempstead, 1996; Vala, Pereira y Ramos, 2000). Los trabajadores que se encuentran en los peldaños socioeconómicos más bajos serían, en función de esta lectura, los más susceptibles a este tipo de competición laboral, debido a que los trabajadores nativos de baja calificación y mal pagos tienen características similares a los recién llegados. En función de esta hipótesis, se esperaría que el ingreso y la educación de los sujetos tengan una fuerte correlación con las actitudes de tolerancia respecto a la inmigración.

Algunos van más allá de esta interpretación y argumentan que aquellos que, más allá de su situación económica, se sienten subjetivamente más desfavorecidos y atribuyen esa desventaja a la presencia de inmigrantes son quienes reaccionan más negativamente frente a estos últimos (Vala, Pereira y Ramos, 2000). Hay quienes también resaltan la necesidad de considerar el contexto para enmarcar estas percepciones de auto-interés económico. Así, el impacto del crecimiento de la fuerza de trabajo vía inmigración 
sería probablemente percibido como menos problemático cuando la economía del país está en expansión, cuando los puestos de trabajo abundan, y cuando hay perspectivas positivas respecto al crecimiento económico (Espenshade y Hempstead, 1996). También apoyando esta teoría se ubica el trabajo de Mayda (2004), el cual analiza desde una perspectiva transnacional la relación entre el nivel de calificación individual y las actitudes a favor de los inmigrantes. En base a esta investigación, la autora encuentra gran variación en la correlación entre ambas variables. Cuanto más rico el país de destino, más positivo es el impacto de la escolaridad en las opiniones favorables sobre los inmigrantes. En cuanto a la calificación individual, la misma aparece correlacionada positivamente con preferencias pro-inmigración en países con un alto ingreso per cápita y negativamente en países con bajo ingreso per cápita.

\section{Factores Culturales: Prejuicio, racismo, xenofobia y sentimientos nacionalistas}

El racismo y los estereotipos étnicos son, de acuerdo a algunas interpretaciones, relevantes en cuanto a la formación de opinión respecto a las políticas públicas. Aquellos que creen en ciertos estereotipos negativos con los que se asocia a algunas minorías tienden a oponerse a políticas que buscan combatir la discriminación racial. Como en los países industrializados las corrientes migratorias están compuestas cada vez en mayor medida por minorías étnicas, las actitudes sobre la inmigración aparecen estrechamente mediadas por la visión de los grupos minoritarios estereotipados negativamente (Burns y Gimpel, 2000). El prejuicio racial hacia las minorías, además, puede darse de manera abierta o solapada (Akrami, Ekehammar y Araya, 2000). 
De acuerdo a Burns y Gimpel (2000), el pensamiento mediado por estereotipos tiene más poder explicativo que el auto-interés económico en lo que respecta a las actitudes respecto a la inmigración. Es más, aunque los autores sostienen que los sectores nativos en los peldaños más bajos del espectro económico suelen oponerse a la inmigración y a los inmigrantes, estas actitudes suelen estar basadas en prejuicios. En consecuencia, aún cuando las condiciones económicas del país mejoren, no es esperable que los nativos acojan cálidamente a los inmigrantes. En un estudio llevado a cabo en la Unión Europea, se muestra además que el prejuicio racial es -junto con el conservadurismo político- uno de los principales predictores de la percepción de amenaza atribuida a la inmigración en los terrenos económico, de seguridad y cultural (Vala Pereira y Ramos, 2000).

Una segunda línea argumental pone atención en el rol que los sentimientos nacionalistas, patrióticos, y hasta el sentido de superioridad nacional tienen en la generación de actitudes antagónicas contra aquellos que no pertenecen a la nación (O'Rourke y Sinnott, 2006). Estas actitudes basadas en ideología serían, por otra parte, más difíciles de modificar que aquellas basadas en intereses, ya que estas últimas podrían ser modificadas a través de políticas complementarias.

En algunos casos, tal como señalan Alba, Rumbaut y Marotz (2005) para el contexto norteamericano, las nociones que prevalecen en la población sobre cómo es la composición étnica y racial del país afectan de manera directa las actitudes de los individuos respecto a la inmigración y los grupos inmigrantes. En particular, de acuerdo a esta investigación, existe una percepción social muy difundida que asigna a las minorías étnicas un peso demográfico mucho mayor que el que en realidad tienen. Y esta percepción distorsionada, a su vez, conlleva el riesgo de una 
mayor intolerancia hacia esos grupos. La idea implícita en esta teoría es que cuanto más "amenazada" se siente la hegemonía blanca en la sociedad, más negativas son las percepciones hacia las minorías raciales o étnicas.

También hay interpretaciones que remiten a la percepción del deseo de los inmigrantes por integrarse a la sociedad de destino. En ese sentido, Zagefka et al. (2007) encuentran evidencia para sostener que cuanto más fuerte es la percepción en la población de que los inmigrantes quieren mantener su propia cultura, más adversas son las actitudes hacia ellos. Paralelamente, cuanto más fuerte es la percepción de que los inmigrantes están abiertos al contacto con la sociedad receptora, menos negativas son las actitudes hacia ellos.

\section{Elementos psicosociales: niveles de satisfacción y felicidad}

La concepción de sentimiento de felicidad sobre la que se asienta este trabajo se refiere al nivel en el cual las personas evalúan positivamente la calidad general de sus vidas como un todo o, lo que es lo mismo, lo que en la literatura se conoce como bienestar subjetivo (Veenhoven, 2000). Si bien existen numerosos estudios que demuestran la correlación existente entre el capital social -en términos de confianza- y la satisfacción de los individuos con sus vidas (por ejemplo, Theurer and Wister, 2010; Sarracino, 2010; Bjørnskov, 2003), la mayoría de estos estudios colocan a la felicidad o satisfacción con la vida como variable dependiente. Es decir, se tiende a explicar la felicidad de los sujetos como producto de múltiples factores entre los que se cuentan las actitudes de relacionamiento respecto de otros genéricos. Sin embargo, la dirección de dicha asociación bien puede plantearse en el sentido inverso, es decir, asumiendo que es el nivel de bienestar subjetivo el 
que determina patrones de relacionamiento con los otros, así como de actitudes públicas (siguiendo, por ejemplo, el planteo de Halman y Luijkx, 2006). Esta es la dirección que retomo para el presente análisis. Pues, en este trabajo parto del supuesto de que la felicidad es de importancia para las actitudes públicas en la medida en que la satisfacción con la vida que a uno le ha tocado vivir tiene implicancias acerca de la apreciación que lo seres humanos tenemos respecto de los otros.

Uno podría suponer que el nivel de satisfacción de un individuo con su propia vida podría tener un impacto en las actitudes de ese mismo individuo acerca de la inmigración. Como es sabido, aquellos individuos que sienten que "no les va bien" en sus vidas tienden a culpar a otros por sus problemas (Espenshade y Hempstead, 1996). Supuestamente, aquellas personas que se sienten más alienadas o insatisfechas estarían menos abiertas a aceptar más inmigrantes. Este grupo de individuos buscarían colocar la responsabilidad de sus problemas en otra parte, y la tendencia es culpar a grupos tales como: los políticos, los pobres y los inmigrantes.

\section{Capital social y confianza}

Diversos estudiosos del capital social señalan una diferenciación entre dos tipos de redes de confianza. Cuando la confianza se establece dentro de redes que miran hacia adentro y tienden a reforzar la homogeneidad del grupo y, por ende, una identidad excluyente estaríamos en presencia de un capital de tipo bonding. Por otro lado, cuando las conexiones tienen lugar entre grupos heterogéneos entre sí, estaríamos frente a un capital de tipo bridging. Si bien ambas clases de capital son capaces de generar valor para los individuos que forman parte de las redes, las características que reviste el valor generado son 
diferentes en uno y otro caso (Putnam, 2000; Brisson, 2009). Numerosos trabajos subrayan los beneficios que el capital de tipo bridging aporta a las minorías en sociedades más amplias (Svendsen, 2006) y las ventajas que el mismo tiene para la cohesión social en general (Scholten y Holzhacker, 2009). Puntualmente, cuando los grupos sociales tienden puentes con otros colectivos diferentes se genera mejor integración social. Por el contrario, cuando un grupo mira predominantemente hacia adentro, es probable que genere hostilidad hacia sectores de afuera (Horiuchi, 2008).

En este último sentido, Berg (2009) ha demostrado cómo el ambiente interpersonal -esto es, el grupo de personas cercanas al individuo y sus opiniones- afecta las actitudes de los individuos acerca de los inmigrantes y la política migratoria. Más aún, más allá del conocimiento o no de inmigrantes de manera directa, los individuos tienen opiniones basadas en nociones transmitidas por sus entornos cercanos. Es decir, los individuos entran en contacto con diferentes actitudes a través de otros individuos que son persuasivos y confiables y, en consecuencia, las adoptan como propias. Por lo tanto, las opiniones sobre inmigración tendrían estrecha relación con la composición del grupo al que pertenece el individuo. En una línea diferente, Hayes y Dowds (2006) destacan que la exposición social previa hacia sujetos inmigrantes -más precisamente, el haber establecido redes de amistad con inmigrantesconstituye el predictor más importante de las actitudes sobre la inmigración.

La relación entre capital social -entendido en términos de confianza- y las actitudes respecto de la inmigración ha sido poco explorada por la literatura. Una excepción es el trabajo de Herreros y Criado (2009), que demuestra que aún controlando los efectos de otras variables (individuales y contextuales), aquellos individuos con un alto nivel de capital social exhiben actitudes más positivas respecto a 
la inmigración que el resto de la población. La literatura sobre capital social ha enfatizado, como vimos, el rol que la confianza tiene para mantener cohesionadas las comunidades. Aquellos individuos con alto nivel de confianza suelen ser mejores ciudadanos y más cooperativos, también tienden a estar más involucrados en la vida en comunidad. Siguiendo a Herreros y Criado (2009), hay razones para creer que el nivel de confianza social tiene un impacto positivo en las actitudes hacia la inmigración. En general, según los autores, aquellos individuos que registran alta confianza social tienen preferencias altruistas en nombre de la comunidad. Sin embargo, y tal como se mencionó anteriormente, una posibilidad pesimista sería que la confianza social esté circunscrita a extranjeros cercanos al origen cultural del que confía.

En este último sentido, puede comprenderse el hallazgo de De la Garza et al. (1991) acerca de la influencia significativa que la "Mexicanidad" (definida como un apego fuerte a las tradiciones culturales mexicanas) tiene en el apoyo a la política inmigratoria en los Estados Unidos. Esta última, cabe aclarar, beneficiaría positivamente a inmigrantes de dicha nacionalidad.

\section{Hipótesis}

En base al análisis de las interpretaciones que en la literatura se ofrecen para intentar dar cuenta del apoyo u oposición hacia las políticas migratorias receptivas, y también a las variables incluidas en la base de datos en la que se basa este estudio, propongo las siguientes hipótesis sobre el tipo de relación esperada entre algunas características de los sujetos o las opiniones que ellos tienen sobre ciertos temas y las actitudes de que los individuos tienen respecto de la inmigración. 
En primer lugar, y de acuerdo a las explicaciones de corte económico prevalecientes en la literatura, sería razonable esperar que el nivel de instrucción formal, la autopercepción de pertenencia de clase, la situación de empleo (empleado/desocupado) y los tipos de tareas desempeñados estén relacionados con las actitudes hacia la política inmigración. En particular, uno esperaría que aquellos individuos con mayor instrucción formal, que se sienten incluidos en estratos sociales más altos, que están empleados y desempeñan tareas no manuales se sientan menos amenazados por la apertura hacia la inmigración $y$, por ende, muestren actitudes de mayor tolerancia. En segundo lugar, es dable esperar que distintos factores de tipo cultural, entre los que se incluyen actitudes de intolerancia hacia la diferencia y sentimientos de nacionalismo, influyan sobre el rechazo hacia la llegada de inmigrantes. Puntualmente, de acuerdo a esta hipótesis, aquellos individuos que valoran los beneficios de una sociedad étnicamente diversa y una actitud menos nacionalista se sentirían menos inclinados a cerrar el ingreso formal de inmigrantes. En tercer lugar, es esperable que a medida que la auto-identificación ideológica tienda más hacia la derecha los individuos tienden a rechazar políticas de inmigración más abiertas. Entonces, las posturas políticas más conservadoras suelen hacer del cierre o la limitación de la inmigración una bandera. En cuarto lugar, considero razonable suponer que a medida que los individuos sienten más satisfacción y felicidad en sus vidas, menos tenderán a culpar a otros y, por ende, más receptivos serán hacia la inmigración. Por último, me gustaría explorar una hipótesis poco trabajada en la literatura, que establece que el nivel de confianza que los individuos tienen en el otro genérico, y en inmigrantes en particular, determina la orientación de sus opiniones respecto de la política inmigratoria. 


\section{Datos y metodología}

El trabajo está basado en bases de datos de la tercera onda de la EMV, relevada en la Argentina en el año 2006. La EMV es un proyecto académico orientado a evaluar los valores socioculturales, morales, religiosos y políticos en diferentes culturas alrededor del mundo. El universo cubierto por la encuesta es la población de hombres y mujeres de 18 años y más. La muestra utilizada fue estratificada en base a dos criterios (geográfico y por tamaño de la población) y al azar por etapas. Primero se seleccionaron aleatoriamente 201 puntos o áreas de muestra dentro de ciudades que también fueron seleccionadas al azar. Aproximadamente cinco entrevistas fueron llevadas a cabo en cada punto muestral. La selección de la unidad muestral final fue realizada de acuerdo a cuotas de edad y sexo preestablecidas. Las cuotas fueron establecidas en base a la distribución de esas variables en cada localidad. Sólo se seleccionaron adultos de 18 años o más, residentes en hogares privados.

La base de datos de la encuesta incluye 1002 casos y provee información sobre un amplio abanico de valores y normas, y resulta de suma utilidad para el presente estudio. Sin embargo, debido a que el foco de este trabajo está puesto en indagar sobre las actitudes de los argentinos sobre las políticas inmigratorias, para los análisis no se mantuvieron los respondentes no argentinos ni aquellos que no manifestaron una respuesta fehaciente a la pregunta sobre actitudes respecto a la inmigración. De esta manera, los casos incluidos en el análisis fueron 939.

Tomo como variable dependiente para este estudio la siguiente pregunta sobre actitudes respecto a la inmigración (que llamaré ACTINM): "Con respecto a gente de otros países que viene a trabajar aquí, ¿cuál de las siguientes alternativas cree ud. que el gobierno debería 
hacer? (1) Permitirle a todos los que quieran venir que vengan; (2) Permitirle a la gente venir en la medida en que haya trabajo disponible; (3) Poner límites estrictos en cuanto al número de extranjeros que pueden venir a nuestro país; (4) Prohibirle a la gente de otros países que vengan aquí; (8) No sabe; (9) No contesta" (ver distribución en cuadro 1). Siguiendo el modelo de Mayda (2004), y a los fines de ganar claridad en la exposición, agrupé las respuestas a la citada pregunta en dos categorías, creando la variable dicotómica "Oposición a la inmigración" (OPINM DUMMY). En primer lugar, los casos cuyas respuestas fueron "No sabe" y "No contesta" fueron excluidos de la muestra. Luego, las respuestas fueron re-codificadas de la siguiente manera: OPINM DUMMY= 1, si ACTINM= 3 ó 4; y OPINM DUMMY=0 si $\mathrm{ACTINM}=1$ ó 2 . En otras palabras, la variable OPINM DUMMY es igual a 1 cuando las opiniones sobre la política migratoria son restrictivas.

\section{Cuadro 1}

"Con respecto a gente de otros países que viene a trabajar aquí, ¿cuál de las siguientes alternativas cree ud. que el gobierno debería hacer?"

\begin{tabular}{|c|c|c|}
\hline Opinión & Frecuencia & Porcentaje \\
\hline $\begin{array}{c}\text { Permitirle a todos los que quieran venir } \\
\text { que vengan }\end{array}$ & 134 & 14.3 \\
\hline $\begin{array}{c}\text { Permitirle a la gente venir en la medida } \\
\text { que haya trabajo disponible }\end{array}$ & 413 & 44.0 \\
\hline $\begin{array}{c}\text { Poner límites estrictos en cuanto al nú- } \\
\text { mero de extranjeros que pueden venir a } \\
\text { nuestro país }\end{array}$ & 333 & 35.5 \\
\hline $\begin{array}{c}\text { Prohibirle a la gente de otros países que } \\
\text { vengan aquí }\end{array}$ & 59 & 6.3 \\
\hline Total & 939 & 100 \\
\hline
\end{tabular}


Las variables independientes que incluí en el modelo, por su parte, fueron agrupadas en seis módulos, a saber: (1) características sociodemográficas; (2) determinantes económicos; (3) factores culturales; (4) ideología; (5) elementos psicosociales; (6) confianza. Las variables y sus categorías se detallan a continuación:

Las variables sociodemográficas abarcan: sexo masculino (dicotómica: 0, 1); edad (intervalar expresada en años); y residencia en ciudad de al menos 100.000 habitantes (dicotómica: 0, 1). Los determinantes económicos incluidos en el modelo son: nivel de instrucción de al menos secundario completo (dicotómica: 0, 1); percepción subjetiva de pertenencia a clase social (nominal: 1 "clase alta"; 2 "clase media alta"; 3 "clase media baja"; 4 "clase trabajadora/obrera"; 5 "clase baja"); condición de desocupado (dicotómica: 0, 1); y naturaleza de tareas desempeñadas, principalmente manuales a principalmente mentales (ordinal: 1 a 10). Los factores culturales considerados incluyen actitudes relativas a prejuicios; racismo y xenofobia; y sentimientos nacionalistas. Puntualmente, las variables son: opinión sobre diversidad étnica, de "erosiona la unidad" a "enriquece la vida" (ordinal: 1 a 10); opinión sobre la frase "cuando los puestos de trabajo escasean, los empleadores deberían admitir antes a los argentinos que a los inmigrantes" (ordinal: 1 "de acuerdo"; 2 "ni de acuerdo ni en desacuerdo"; 3 "en desacuerdo"); y "hasta qué punto está ud. orgulloso de ser argentino" (ordinal: de 1 "muy orgulloso" a 4 "poco orgulloso"). Tomé en consideración una variable ideológica, a saber: dónde se colocaría ud. en escala de izquierda a derecha (ordinal, con un rango que va de 1 representando la extrema izquierda a 10 simbolizando la extrema derecha). Los elementos psicosociales incorporados en el análisis se refieren a los niveles de satisfacción con la vida. Específicamente, tomé de la base de datos 
la siguiente variable: y "en general, cuán feliz es usted" (ordinal: de 1 "muy feliz" a 4 "nada feliz"). ${ }^{4}$ Por último, el modelo incluye las siguientes variables vinculadas a sentimientos de confianza: identificación con la frase "cree Ud. que la mayoría de la gente se aprovecharía de ud. si tuviera la oportunidad o por el contrario lo trataría de manera justa" (ordinal: 1 a 10); y confianza en gente de otra nacionalidad (ordinal: de 1 "confía plenamente" a 4 "no confía nada").

Cuadro 2. Variables incluidas en el análisis multivariado de regresión logística (estadísticas descriptivas luego de la imputación múltiple, base agregada)

\begin{tabular}{|c|c|c|}
\hline Variable & Media & Rango \\
\hline Oposición a la inmigración & 0.42 & $0-1$ \\
\hline Varón & 0.48 & $0-1$ \\
\hline Edad & 42.56 & $18-88$ \\
\hline Al menos secundario completo & 0.50 & $0-1$ \\
\hline Residencia en ciudad - 100.000 habs. & 0.69 & $0-1$ \\
\hline Auto-identificación clase baja o trabajadora & 3.47 & $1-5$ \\
\hline Condición de desocupado & 0.69 & $0-1$ \\
\hline Tareas manuales vs. Mentales & 4.16 & $1-10$ \\
\hline Opinión sobre diversidad étnica & 7.10 & $1-10$ \\
\hline Empleadores deberían contratar argentinos & 1.47 & $1-3$ \\
\hline Orgullo nacionalidad & 1.47 & $1-4$ \\
\hline Auto-posicionamiento en escala política & 5.55 & $1-10$ \\
\hline Sentimiento de felicidad & 1.83 & $1-4$ \\
\hline Mayoría trata de sacar provecho vs. Trato justo & 4.96 & $1-10$ \\
\hline Confianza en gente de otra nacionalidad & 2.38 & $1-4$ \\
\hline
\end{tabular}

En modelos que probamos antes habíamos incluido también las variables “¿Hasta qué punto está ud. satisfecho con su vida?” y “Satisfacción con la situación financiera de su hogar", pero decidimos sacarlas del modelo ya que presentaban problemas de multicolinearidad con la variable sobre percepción de felicidad. 
Debido a que la variable dependiente es dicotómica, la forma apropiada de análisis es alguna forma de análisis log-linear en lugar de la regresión OLS. Utilicé, en consecuencia, le técnica de regresión logística (logit) para producir una ecuación mediante la cual se estiman las probabilidades de rechazo a las políticas de inmigración favorables. Este procedimiento me permitió estimar las probabilidades de rechazo en un número de circunstancias diferentes. Apliqué, entonces, un análisis de regresión logística en la base de datos agregada (incluyendo las 5 imputaciones de datos faltantes), utilizando el rechazo a la política inmigratoria abierta como variable dependiente y 14 variables independientes distribuidas en 6 modelos diferentes. Para dicho análisis, utilicé el programa de análisis estadístico SPSS.

\section{Resultados}

En una comparación realizada entre 44 países (tanto industrializados como no industrializados) basados en datos de la tercera onda de la EMV, realizada en 1995-1997, la Argentina aparece como un país de tolerancia media respecto de las políticas migratorias (Mayda, 2004: 55). En dicho relevamiento, la Argentina registraba un 59\% de actitudes abiertas y tolerantes respecto de la política inmigratoria a adoptar por el gobierno, mientras la media de actitudes positivas para todo el grupo de países era del 53\%. En ese listado, además, la Argentina aparece en la posición número 16 entre 44 en cuanto a la tolerancia mostrada por la opinión pública respecto de la inmigración. Es decir que la Argentina se ubica cómodamente en 
la mitad de países con actitudes menos restrictivas hacia la recepción de inmigrantes para trabajar. ${ }^{5}$

El nivel de apoyo hacia las políticas inmigratorias gubernamentales abiertas prácticamente no ha variado en las últimas tres mediciones que se llevaron a cabo en la Argentina. En la medición llevada a cabo en 1995, el 59\% de las actitudes eran a favor de políticas inmigratorias abiertas; en 1999, el valor fue del 56\%, y en la tercera, desarrollada en 2006, las actitudes de apertura hacia la inmigración nuevamente se ubicaron en el $59 \%$.

Más allá de eso, poco se sabe acerca de quiénes se oponen a la inmigración y por qué. Nuestros análisis basados en regresiones logísticas múltiples dan respaldo a algunas de las explicaciones generalmente aceptadas para otros contextos nacionales, al mismo tiempo que contradicen algunos de los resultados esperados. Tal como se ve en el Cuadro 3, ajusté un total de seis modelos a los datos. ${ }^{6}$

5 De acuerdo al estudio de Mayda (2004), la proporción de actitudes positivas sobre la inmigración en la Argentina (59\%) era más alta que en otros países tradicionalmente receptores de inmigrantes como los Estados Unidos (35\%), Suecia (41\%), o Australia (53\%), pero no tan alta como en Brasil (61\%) y Uruguay (69\%).

${ }_{6} \quad$ Cabe aclarar que, a fin de simplificar la exposición, el modelo que se presenta aquí está basado en los datos agregados resultantes de las 5 imputaciones de valores faltantes. Las relaciones que se presentan aquí se mantienen a grandes rasgos en las cinco bases de datos simuladas resultantes de la imputación de valores faltantes. Dichas bases de datos se incluyen en el anexo. 


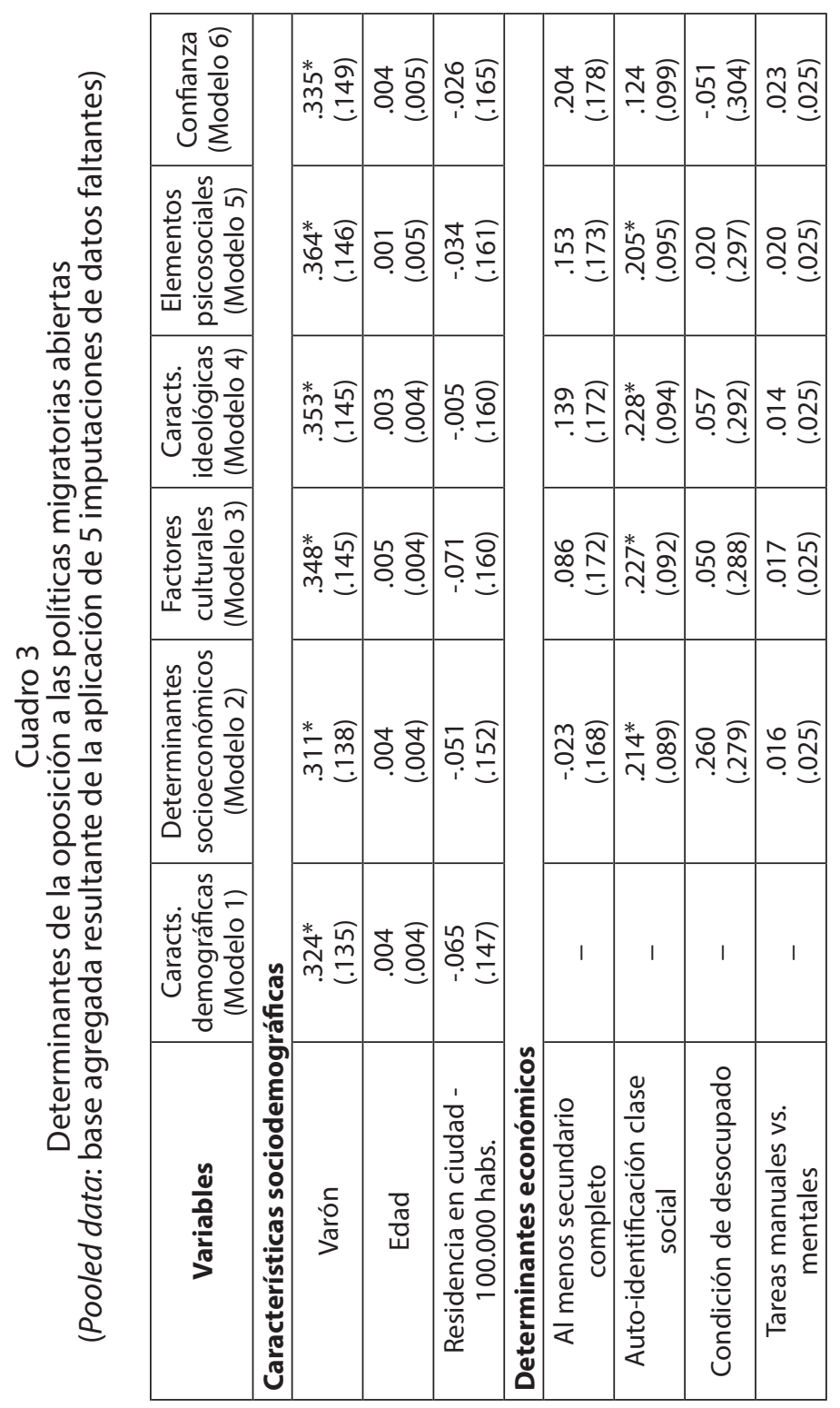




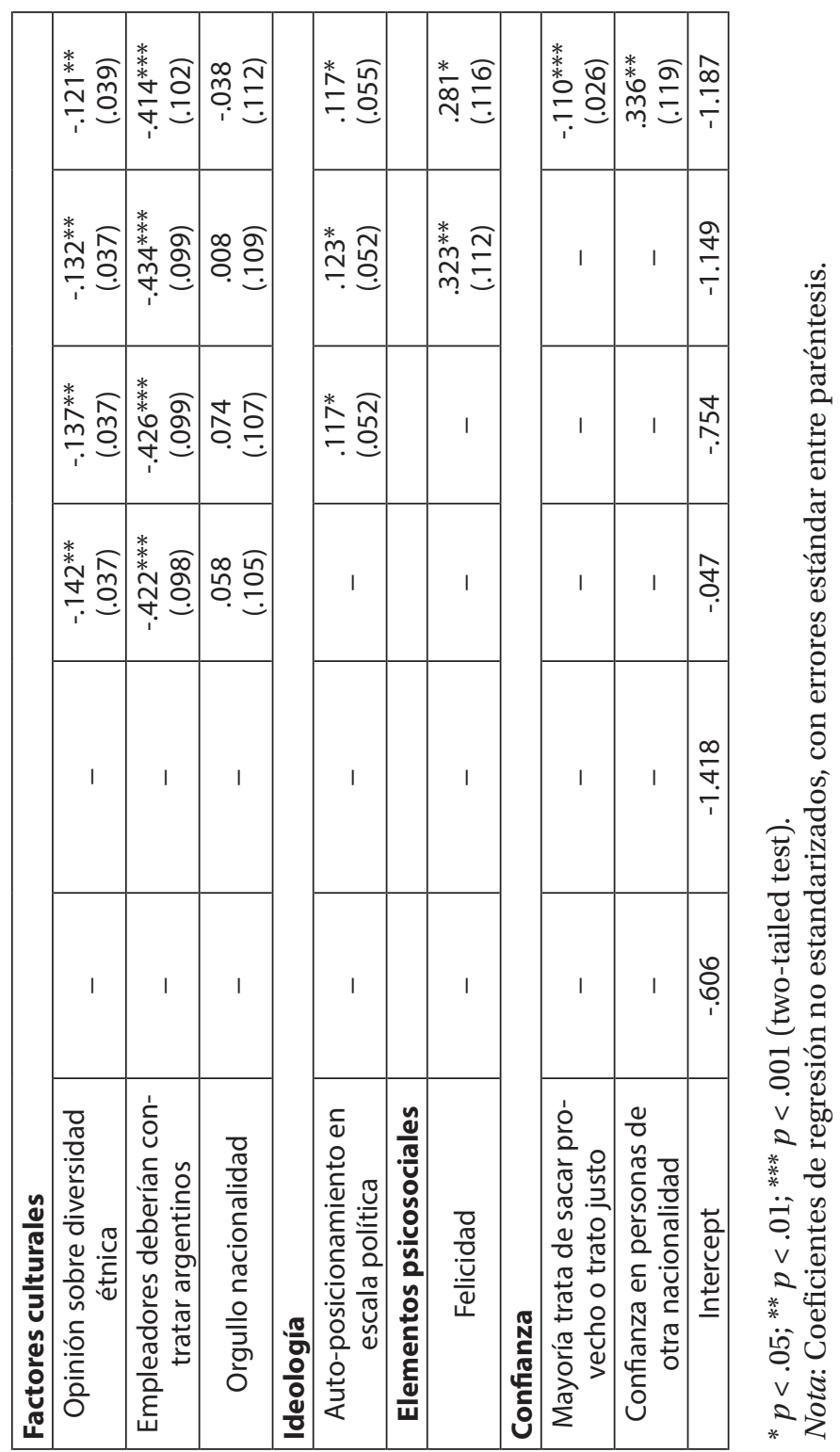


En el modelo 1 surge que de las variables demográficas consideradas, sólo el sexo masculino tiene un impacto significativo y positivo sobre actitudes de oposición a la inmigración. Por su parte, ni la edad ni el tamaño de la localidad de residencia aparecen significativamente vinculados a las opiniones sobre la política migratoria. En otras palabras, los varones tienden a expresar actitudes más cerradas respecto de la inmigración en comparación con las mujeres. Esa relación permanece significativa y en la misma dirección aún controlando por otras variables en los modelos sucesivos. ${ }^{7}$ Contradiciendo lo esperado, la residencia en ciudades más grandes no aparece negativamente asociada con las posturas más restrictivas respecto a la inmigración.

El modelo 2, por su parte, pone a prueba mi primera hipótesis, a saber: que aquellos segmentos de la población en situación más vulnerable -con menor calificación y educación- exhiben actitudes más anti-inmigratorias en virtud de que se sienten más amenazados en sus intereses por los recién llegados. En función de ello, se anticipaba que el nivel de instrucción formal, la autopercepción de pertenencia de clase, la situación de empleo (empleado/ desocupado) y los tipos de tareas desempeñados estuvieran relacionados con las actitudes más tolerantes hacia la inmigración. Con respecto al nivel de instrucción, el análisis de los datos muestra que los sujetos con al menos secundario completo no presentan una correlación significativa con las actitudes respecto de la política inmigratoria, desechando la presunción de que a mayor educación, más abiertas serían las actitudes hacia la inmigración. Algo

Si bien son escasos los estudios sobre las diferencias de género en materia de preferencia sobre políticas pública, algunos estudios en otros países señalan que las mujeres tienden a sostener posiciones más tolerantes (Sotelo, 1999) y opiniones políticas más progresistas (Crowder-Meyer, 2007) que los varones. 
diferente ocurre con el comportamiento de la variable autoidentificación de clase; si bien la base de datos no cuenta con información sobre ingresos, la variable considerada como proxy -esto es, autoidentificación de clase- aparece asociada con las opiniones sobre la política inmigratoria. Concretamente, cuanto más baja es la clase con la que se identifican los individuos, más aumenta el rechazo hacia las políticas inmigratorias abiertas. Esta relación se mantiene significativa en algunos modelos parciales, pero deja de ser significativa en el modelo completo. La condición de desocupado, o la naturaleza de las tareas desempeñadas (manual vs. mentales), por su parte, no aparecen relacionadas con las preferencias en materia de política de inmigración. Es decir, la hipótesis que señala que cuanto más abajo uno se encuentre en la escala de estratificación social, más amenazado se sentiría por la eventual llegada de nuevos individuos por visualizarlos como competidores directos, encuentra poco sustento en el caso bajo estudio. Este hallazgo es importante teniendo en cuenta la fuente presencia que tienen en la literatura comparada las explicaciones de corte económico a la hora de dar cuenta de las actitudes respecto de la inmigración.

En el modelo 3 someto a verificación empírica la segunda hipótesis, que establece que factores de tipo cultural tales como actitudes de intolerancia hacia la diferencia y sentimientos de nacionalismo, influyen sobre el rechazo hacia la llegada de inmigrantes. El comportamiento de las variables de corte "cultural" que forman parte del análisis es dispar. Por un lado, y tal como se había anticipado, aquellos que tienen una opinión más negativa sobre el valor de la diversidad étnica para la sociedad tienden a sostener visiones más anti-inmigración. Esta relación se mantiene significativa y en la misma dirección incluso cuando se añaden otras variables de control. La otra relación que se mantiene significativa, aun al incluir otros módulos de 
variables al modelo es la existente entre la opinión sobre la frase "cuando los puestos de trabajo escasean, los empleadores deberían admitir antes a los argentinos que a los inmigrantes" y la actitud respecto a las políticas de inmigración. Dicho en otros términos, aquellos que sostienen que en época de crisis los empleadores deberían priorizar mano de obra local tienden a oponerse más a la inmigración. Esta variable, si bien tiene un contenido económico, contiene un fuerte componente cultural -basado en la visión respecto al corte tajante entre un "nosotros" y un "tercero" imaginario, basados en la nacionalidad-. Contrariamente a lo esperado, sin embargo, los sentimientos nacionalistas no aparecen significativamente asociados con las actitudes respecto a la inmigración.

La tercera hipótesis -que establece que controlando los efectos de otras variables, a medida que la autoidentificación ideológica tienda más hacia la derecha los individuos tienden a rechazar en mayor grado políticas de inmigración más abiertas- es presentada en el Modelo 4. La evidencia presentada me permite sostener esa hipótesis. Es decir que, en el plano ideológico, tal como ocurre en otras temáticas públicas, aquellos que se identifican más hacia la derecha en el espectro político son menos tolerantes respecto a la inmigración que aquellos que se reconocen políticamente más hacia la izquierda.

En cuanto a los elementos psicosociales considerados, tal como se puede observar en el Modelo 5, aquellos que se autorreconocen como menos felices tienden a tener actitudes más negativas hacia la inmigración. Este patrón responde a lo esperado, en la medida en que cuando uno se siente más feliz menos hostilidad tiende a mostrar respecto a terceros.

Por último, basándome en las hipótesis con eje en la confianza, preveía que aquellos con más confianza en otros que no conocen tuvieran actitudes más abiertas respecto 
a la inmigración. Como se puede observar en el Modelo 6 , los coeficientes relacionados a la confianza social son significativos y se comportan en la dirección esperada. Por un lado, para los que confían poco en los demás, es decir, los que sienten que la mayoría trata de aprovecharse de ellos, las actitudes sobre la inmigración son más restrictivas. En otras palabras, aquellos que se sienten más globalmente amenazados por otros a quienes no conocen prefieren tener una política estatal más cerrada respecto a eventuales nuevos migrantes. También fue incluida en este modelo una variable que se refiere puntualmente a la confianza en personas de otra nacionalidad, y tal como se anticipaba, cuanto menos se confía en individuos de diferentes nacionalidades, más opuestas son las actitudes respecto a una política inmigratoria abierta.

En general, el rechazo a las políticas inmigratorias abiertas no parece estar basado en motivaciones de tipo económico, como se sostiene en numerosos estudios realizados en otros países. Por el contrario, tal como se desprende de nuestro análisis, dicho rechazo parece estar más asociado a factores culturales, ideológicos, psicosociales y de confianza. En síntesis, puede decirse que quienes más se oponen a la inmigración tienden a ser varones, menos tolerantes a la diversidad, con posturas más conservadoras, menos felices en sus vidas, y con actitudes de mayor desconfianza hacia terceros desconocidos o terceros diferentes.

\section{Discusión}

En primer lugar, a nivel comparado, la actitud de oposición respecto a la inmigración en la Argentina no es tan acentuada como en otros países. Esto significa que, si bien como señala la literatura hay un complejo entramado de discursos y prácticas discriminatorias que estigmatizan a 
los inmigrantes desde diferentes ámbitos (tanto desde el estado en sus diferentes niveles, como en los medios de comunicación, en el sistema educativo y en interacciones cotidianas), en términos comparados y en base a la actitud respecto de las políticas migratorias no podemos señalar a la sociedad argentina como un colectivo fuertemente xenófobo.

En cuanto a las variables que dan cuenta de las actitudes restrictivas respecto a la política inmigratoria a seguir, uno de los hallazgos más fuertes de este trabajo es que las explicaciones económicas que aluden a la percepción de amenaza a la auto satisfacción económica no parecen, en general, tener cabida en la Argentina. Al contrario, aquellos factores con poder explicativo para dar cuenta de las opiniones sobre la política inmigratoria tienden a estar más centrados en la confianza respecto de terceros, así como en aspectos culturales, psicosociales e ideológicos.

Como señalan algunos autores, esto plantea un problema para la política pública, ya que es más fácil modificar intereses (mediante políticas complementarias) que valores. Para el caso presentado aquí, son básicamente nociones acerca del otro genérico, percepciones de sí mismo (felicidad), y actitudes acerca de cómo debería funcionar el mundo (ideología) los que tienen un impacto sensible sobre la manera en la que el estado debería manejarse respecto de la eventual llegada de inmigrantes de otros países.

En vistas a contribuir a la paz social, esto plantea a nivel de políticas públicas un desafío fundamentalmente educativo, donde se impone la necesidad de desestigmatizar a los inmigrantes. Por lo demás, los patrones culturales son constructos que adoptan lógicas propias y el cambio cultural es, como es sabido, una cuestión de largo plazo. 


\section{Referencias bibliográficas}

Akrami, Nazar, Bo Ekehammar y Tadesse Araya (2000), "Classical and Modern Racial Prejudice: A Study of Attitudes toward Immigrants in Sweden", European Journal of Social Psychology 30, pp. 521-532.

Alba, Richard, Ruben G. Rumbaut y Karen Marotz (2005), “A Distorted Nation: Perceptions of Racial/Ethnic Group Sizes and Attitudes Toward Immigrants and Other Minorities", Social Forces 84(2), pp. 901-919.

Berg, Justin Allen (2009), “'Core Networks and Whites' Attitudes toward Immigrants and Immigration Policy", Public Opinion Quarterly 73(1), pp. 7-31.

Bjørnskov, Christian (2003), "The Happy Few: Cross-Country Evidence on Social Capital and Life Satisfaction", Kyklos 56(1), pp. 3-16.

Brisson, Daniel (2009), “Testing the Relationship of Formal

Bonding, Informal Bonding, and Formal Bridging Social Capital on Key Outcomes for Families in LowIncome Neighborhoods", Journal of Sociology \& Social Welfare XXXVI(1), pp. 167-183.

Boomgaarden, Hajo G. y Rens Vliegenthart (2009), "How

News Content Influences Anti-immigration Attitudes: Germany, 1993-2005", European Journal of Political Research 48, pp. 516-542.

Burns, Peter y James G. Gimpel (2000), “Economic Insecurity, Prejudicial Stereotypes, and Public Opinion on Immigration Policy", Political Science Quarterly 115(2), pp. 201-225.

Crowder-Meyer, Melody (2007), "Gender Differences in

Policy Preferences and Priorities", ponencia presentada en la Reunión Anual de la Midwest Political Science Association en Chicago.

De la Garza, Rodolfo O., Jerry L. Polinard, Robert D. Wrinkle y Tomás Longoria (1991), "Understanding Intra-Ethnic 
Attitude Variations: Mexican Origin Population Views of Immigration", Social Science Quarterly 72(2), 379-387. Diamond, Jeff (1998), "African-American Attitudes towards United States Immigration Policy", International Migration Review 32(2), pp. 451-470.

Espenshade, Thomas y Katherine Hempstead (1996), "Contemporary American Attitudes Toward U.S. Immigration", International Migration Review XXX(2), pp. 535-570.

Esses, Victoria M., John F. Dovidio y Gordon Hodson (2002),

"Public Attitudes Toward Immigration in the United States and Canada in Response to the September 11, 2001 'Attack on America"', Analyses of Social Issues and Public Policy 2(1), pp. 69-85.

Gallinati, Carla (2008), “¿Inmigrantes o Ciudadanos? La Construcción del 'Verdadero' Modelo de Política Migratoria en el Marco de la 'Patria Grande', Pensares 5, pp. 479-500.

Grimson, Alejandro (2005), Relatos de la Diferencia y la Igualdad. Los bolivianos en Buenos Aires, Buenos Aires, EUDEBA.

(2006), "Nuevas Xenofobias, Nuevas Políticas Étnicas en Argentina." En A. Grimson y E. Jelin, Migraciones Regionales hacia la Argentina:Diferencia, Desigualdad y Derechos, Buenos Aires, Prometeo Libros, pp. 69-97. Halman, Loek y Ruud Luijkx (2006), "Social Capital in Contemporary Europe: Evidence from the European Social Survey", Journal of Social Science 5(1), pp. 65-90. Hayes, Bernadette C. y Lizanne Dowds (2006), "Social Contact, Cultural Marginality or Economic SelfInterest? Attitudes Towards Immigrants in Northern Ireland", Journal of Ethnic and Migration Studies 32(3), pp. 455-476.

Herreros, Francisco y Henar Criado (2009), "Social Trust, Social Capital and Perceptions of Immigration", Political Studies 57, pp. 337-355. 
Horiuchi, Shiro (2008), "Affiliative Segregation of Outsiders from a Community: Bonding and Bridging Social Capital in Hachimori-cho, Japan", International Journal of Japanese Sociology 17, pp. 91-100.

Joseph, Galen (2000), “Taking Race Seriously: Whiteness in Argentina's National and Transnational Imaginary", Identities 7(3), pp. 333-371.

Lupo, Giampiero (2010), "Is Immigration Detrimental for Social Trust in the European Union? A Three-Level Model of Cultural Heterogeneity and Citizenship Regime as Social Capital Predictors", International Journal of Social Inquiry 3(1), pp. 67-96.

Mayda, Anna Maria (2004), "Who Is Against Immigration? A Cross-Country Investigation of Individual Attitudes toward Immigrants", IZA Discussion Paper No 1115, Bonn, IZA.

Monner Sans, Ana Inés (2005), “Marcadores de Valor y Disvalor en Situaciones de Contacto Sociocultural: Perpepción y Expresión de la Diferencia a través del Discurso" en E. Domenech (comp.) Migraciones Contemporáneas y Diversidad Cultural en la Argentina, Córdoba, Universidad Nacional de Córdoba, pp. 97-130. Nadali, Débora Betrisey (2007), “Inmigración y Discriminación en la Frontera Argentino-Paraguaya", Migraciones Internacionales 4(1), pp. 141-164.

O'Rourke, Kevin H. y Richard Sinnott (2006), “The Determinants of Individual Attitudes Towards Immigration", European Journal of Political Economy 22, pp. 838-861.

Oteiza, Enrique, Susana Novick y Roberto Aruj (2000), Inmigración y Discriminación: Políticas y Discursos, Buenos Aires, Trama Editorial/Prometeo Libros.

Pacecca, María Inés y Corina Courtis (2008), "Inmigración Contemporánea en Argentina: Dinámicas y Políticas”, CEPAL - Serie Población y desarrollo No 84. 
Pevnick, Ryan (2009), "Social Trust and the Ethics of Immigration Policy", The Journal of Political Philosophy 17(2), pp. 146-167.

Putnam, Robert (2000), Bowling Alone: The Collapse and Revival of American Community, Londres, Simon \& Schuster.

Rose, Rodericky Mark Fraser (2008) "A Simplified Framework for Using Multiple Imputation in Social Work Research", Social Work Research 32(3): 171-178.

Sarracino, Francesco (2010), "Social Capital and Subjective Well-Being Trends: Comparing 11 Western European Countries", Journal of Socio-Economics 39(4), pp. 482-517.

Scheve, Kenneth F. y Matthew J. Slaughter (2001), "Labor Market Competition and Individual Preferences over Immigration Policy", The Review of Economics and Statistics 83(1), pp. 133-145.

Scholten, Peter y Ronald Holzhacker (2009), "Bonding, Bridging and Ethnic Minorities in the Netherlands: Changing Discourses in a Changing Nation", Nations and Nationalism 15(1), pp. 81-100.

Simon, Rita J. y James P. Lynch (1999), "A Comparative

Assessment of Public Opinion toward Immigrants and Immigration Policies", International Migration Review 33(2): 455-467.

Sotelo, María José (1999), "Gender Differences in Political Tolerance among Adolescents", Journal of Gender Studies 8(2), pp. 211-217.

Svendsen, Gunnar L. H. (2006), "Studying Social Capital in situ: A Qualitative Approach", Theory and Society 35(1), pp. 39-70.

Theurer, Kristine and Andrew Wister (2010), "Altrustic Behaviour and Social Capital as Predictors of WellBeing among Older Canadians", Ageing and Society 30(1), pp. 157-181. 
Vala, Jorge, Cícero Pereira y Alice Ramos (2006), "Racial Prejudice, Threat Perception and Opposition to Immigration: A Comparative Analysis", Portuguese Journal of Social Science 5(2), pp. 119-139.

Veenhoven, Ruut (2000), "Freedom and Happiness: A Comparative Study in Forty-four Nations in the Early 1990s", en Ed Diener y Eunkook M. Suh (Eds.), Culture and Subjective Well-Being, Cambridge, MA, MIT Press, pp. 257-288.

Zagefka, Hanna, Rupert Brown, Murielle Broquard y Sibel Leventoglu Martin (2007), "Predictors and Consequences of Negative Attitudes toward Immigrants in Belgium and Turkey: The Role of Acculturation Preferences and Economic Competition", British Journal of Social Psychology 46, pp. 153-169

\section{Apéndice}

\section{a. Imputación de valores}

La imputación de valores para las variables independientes con respuestas "no sabe", "no contesta", o "no aplica" se efectuó usando el método de imputaciones múltiples (IM). En primer lugar, realicé un análisis de los valores faltantes usando el método de Little's MCAR mediante el cual me fue posible comprobar que los datos faltantes no son faltantes completamente al azar. En ese caso, la estrategia de IM aparece como la menos sesgada y, por ende, recomendada por la literatura (ver, por ejemplo, Rose y Fraser, 2008). Puntualmente, los valores faltantes fueron sustituidos por valores derivados de distribuciones de probabilidad condicionales simuladas creando, en consecuencia, cinco versiones de la base de datos. 


\begin{tabular}{|c|c|c|c|c|c|c|c|c|c|}
\hline 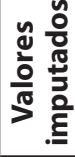 & $\stackrel{\stackrel{\sim}{N}}{N}$ & $\stackrel{n}{n}$ & $\stackrel{\mathfrak{n}^{\prime}}{\wedge}$ & $\stackrel{\text { n }}{\mp}$ & ৪ & $\stackrel{P}{\underline{m}}$ & 우 & $\stackrel{\text { Ln }}{\sim}$ & 유 \\
\hline 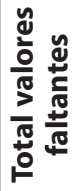 & 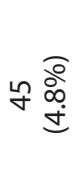 & 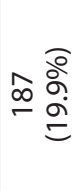 & 守总 & 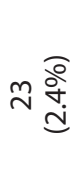 & 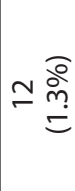 & 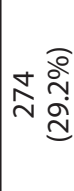 & 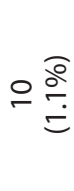 & ñ & 응 \\
\hline 은 $\frac{\sqrt{5}}{\frac{\pi}{0}}$ & ' & 守 & ' & ' & ' & 1 & ' & I & 1 \\
\hline 용 & 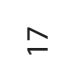 & 으 & $\underset{\mathcal{I}}{\sim}$ & in & & $\stackrel{\text { ㅁ }}{\sim}$ & 0 & $\hat{f}$ & $\curvearrowright$ \\
\hline 욜 & $\stackrel{\infty}{\sim}$ & $\stackrel{\infty}{\sim}$ & in & $\infty$ & $\infty$ & $\stackrel{\infty}{\sim}$ & $\nabla$ & 0 & $\hat{\imath}$ \\
\hline$\frac{\frac{0}{0}}{\frac{0}{\frac{0}{2}}}$ & 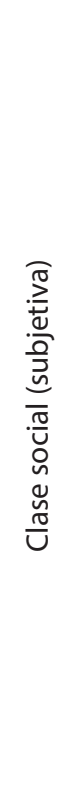 & 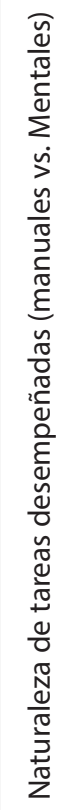 & 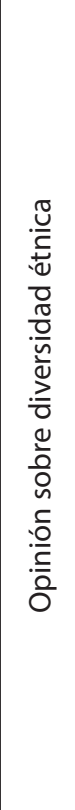 & 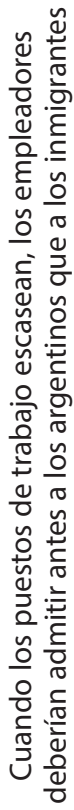 & 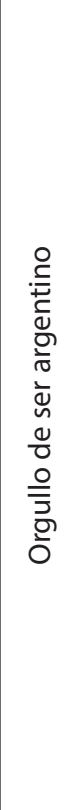 & 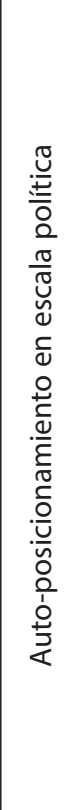 & 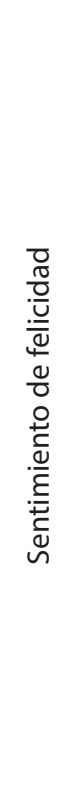 & 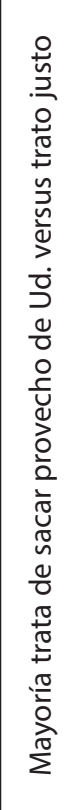 & 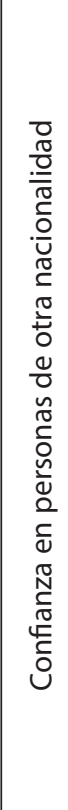 \\
\hline
\end{tabular}




\section{b. Determinantes de la oposición a las} políticas migratorias abiertas

Coeficientes y errores estándar correspondientes a 5 simulaciones- modelos completos

\begin{tabular}{|c|c|c|c|c|c|}
\hline Variables & \begin{tabular}{|c|} 
Simulación \\
1
\end{tabular} & \begin{tabular}{|c|} 
Simulación \\
2
\end{tabular} & \begin{tabular}{|c|} 
Simulación \\
3
\end{tabular} & $\begin{array}{c}\text { Simulación } \\
4\end{array}$ & $\begin{array}{c}\text { Simulación } \\
5\end{array}$ \\
\hline \multicolumn{6}{|c|}{ Características sociodemográficas } \\
\hline Varón & \begin{tabular}{|l|}
$.341^{*}$ \\
$(.145)$ \\
\end{tabular} & $\begin{array}{l}.375^{*} \\
(.145)\end{array}$ & $\begin{array}{l}.294^{*} \\
(.147)\end{array}$ & $\begin{array}{l}.332^{*} \\
(.146)\end{array}$ & $\begin{array}{l}.332^{*} \\
(.146)\end{array}$ \\
\hline Edad & $\begin{array}{l}.002 \\
(.004)\end{array}$ & $\begin{array}{l}.004 \\
(.004)\end{array}$ & $\begin{array}{l}.003 \\
(.005)\end{array}$ & $\begin{array}{l}.004 \\
(.004)\end{array}$ & $\begin{array}{l}.006 \\
(.004)\end{array}$ \\
\hline $\begin{array}{c}\text { Residencia en ciudad - } \\
100.000 \text { habs. }\end{array}$ & \begin{tabular}{|l}
-.017 \\
$(.163)$ \\
\end{tabular} & $\begin{array}{l}-.011 \\
(.164)\end{array}$ & $\begin{array}{l}-.052 \\
(.167)\end{array}$ & $\begin{array}{l}-.015 \\
(.164) \\
\end{array}$ & $\begin{array}{l}-.033 \\
(.164) \\
\end{array}$ \\
\hline \multicolumn{6}{|c|}{ Determinantes económicos } \\
\hline $\begin{array}{c}\text { Al menos secundario } \\
\text { completo }\end{array}$ & \begin{tabular}{|c|}
.200 \\
$(.176)$ \\
\end{tabular} & $\begin{array}{r}.235 \\
(.174) \\
\end{array}$ & $\begin{array}{l}.171 \\
(.178)\end{array}$ & $\begin{array}{c}.193 \\
(.176)\end{array}$ & $\begin{array}{c}.223 \\
(.174) \\
\end{array}$ \\
\hline $\begin{array}{c}\text { Auto-identificación cla- } \\
\text { se baja o trabajadora }\end{array}$ & $\begin{array}{l}.141 \\
(.092)\end{array}$ & $\begin{array}{l}.106 \\
(.092)\end{array}$ & $\begin{array}{l}.170 \\
(.094)\end{array}$ & $\begin{array}{l}.100 \\
(.095)\end{array}$ & $\begin{array}{l}.105 \\
(.095)\end{array}$ \\
\hline $\begin{array}{l}\text { Condición de } \\
\text { desocupado }\end{array}$ & $\begin{array}{l}-.021 \\
(.293)\end{array}$ & $\begin{array}{l}-.177 \\
(.283)\end{array}$ & $\begin{array}{l}.054 \\
.(293)\end{array}$ & $\begin{array}{l}-.050 \\
(.291)\end{array}$ & $\begin{array}{l}-.060 \\
(.291)\end{array}$ \\
\hline $\begin{array}{c}\text { Tareas manuales vs. } \\
\text { mentales }\end{array}$ & $\begin{array}{l}.018 \\
(.024)\end{array}$ & $\begin{array}{l}.023 \\
(.024)\end{array}$ & $\begin{array}{c}.033 \\
(.025) \\
\end{array}$ & $\begin{array}{l}.022 \\
(.024) \\
\end{array}$ & $\begin{array}{l}.018 \\
(.025)\end{array}$ \\
\hline \multicolumn{6}{|l|}{ Factores culturales } \\
\hline $\begin{array}{c}\text { Opinión sobre diversi- } \\
\text { dad étnica }\end{array}$ & $\begin{array}{c}-.100^{* * *} \\
(.027) \\
\end{array}$ & $\begin{array}{l}-.086^{* *} \\
(.027) \\
\end{array}$ & $\begin{array}{c}-.141^{* * *} \\
(.028) \\
\end{array}$ & $\begin{array}{c}-138^{* * *} \\
(.027) \\
\end{array}$ & $\begin{array}{l}-.141^{* * *} \\
(.027) \\
\end{array}$ \\
\hline $\begin{array}{c}\text { Empleadores deberían } \\
\text { contratar argentinos }\end{array}$ & $\begin{array}{c}-.423^{* * *} \\
(.100) \\
\end{array}$ & $\begin{array}{c}-.440^{* * *} \\
(.100) \\
\end{array}$ & $\begin{array}{c}-.407^{* * *} \\
(.100) \\
\end{array}$ & $\begin{array}{l}-.406^{* * *} \\
(.100) \\
\end{array}$ & $\begin{array}{l}-.393^{* * * *} \\
(.101) \\
\end{array}$ \\
\hline Orgullo nacionalidad & $\begin{array}{l}-.029 \\
(.110) \\
\end{array}$ & $\begin{array}{l}-.021 \\
(.111) \\
\end{array}$ & $\begin{array}{l}-.038 \\
(.112) \\
\end{array}$ & $\begin{array}{l}-.066 \\
(.111) \\
\end{array}$ & $\begin{array}{l}-.033 \\
(.111) \\
\end{array}$ \\
\hline \multicolumn{6}{|l|}{ Ideología } \\
\hline $\begin{array}{c}\text { Auto-posicionamiento } \\
\text { en escala política }\end{array}$ & $\begin{array}{l}.142^{* * *} \\
(.039) \\
\end{array}$ & $\begin{array}{l}.123^{* *} \\
(.040)\end{array}$ & $\begin{array}{l}.158^{* * *} \\
(.040)\end{array}$ & $\begin{array}{l}.081^{*} \\
(.040)\end{array}$ & $\begin{array}{l}.084^{*} \\
(.040)\end{array}$ \\
\hline \multicolumn{6}{|c|}{ Elementos psicosociales } \\
\hline Felicidad & $\begin{array}{l}.317^{* *} \\
(.112) \\
\end{array}$ & $\begin{array}{l}.248^{*} \\
(.111)\end{array}$ & $\begin{array}{l}.286^{*} \\
(.114)\end{array}$ & $\begin{array}{l}.268^{*} \\
(.112)\end{array}$ & $\begin{array}{l}.285^{*} \\
(.113)\end{array}$ \\
\hline \multicolumn{6}{|l|}{ Confianza } \\
\hline $\begin{array}{l}\text { Mayoría trata de sacar } \\
\text { provecho o trato justo }\end{array}$ & $\begin{array}{c}-.103^{* * *} \\
(.025) \\
\end{array}$ & $\begin{array}{l}-.114^{* * *} \\
(.025)\end{array}$ & $\begin{array}{c}-.118^{* * *} \\
(.025)\end{array}$ & $\begin{array}{l}-.108^{* * *} \\
(.025)\end{array}$ & $\begin{array}{c}-.107 * * * \\
(.025)\end{array}$ \\
\hline $\begin{array}{l}\text { Confianza en personas } \\
\text { de otra nacionalidad }\end{array}$ & $\begin{array}{l}.242^{* *} \\
(.092)\end{array}$ & $\begin{array}{l}.386^{* * *} \\
(.093)\end{array}$ & $\begin{array}{l}.299^{* *} \\
(.095)\end{array}$ & $\begin{array}{l}.345^{* * *} \\
(.092)\end{array}$ & $\begin{array}{l}.407^{* * *} \\
(.093)\end{array}$ \\
\hline Intercept & -1.327 & -1.477 & -1.255 & -.773 & -1.102 \\
\hline Pseudo-R2 & .159 & .166 & .193 & .172 & .184 \\
\hline
\end{tabular}

${ }^{*} p<.05$; $^{* *} p<.01$; $^{* * *} p<.001$ (two-tailed test). 